\title{
COMMUNITIES OF EPIGEIC BEETLES IN TREE LINE FROM MONTANE SPRUCE FOREST TO SECONDARY MEADOW IN THE DIFFERENT STAGE OF THE FOREST DECLINE IN THE AREA OF MODRAVA (BOHEMIAN FOREST, CZECH REPUBLIC)
}

\author{
JAROSLAV BOHÁČ, KAREL MATĚJKA
}

Faculty of Agriculture, University of South Bohemia in České Budějovice, Studentská 13, 37005 České Budějovice, Czech Republic; e-mail: jardaboh@seznam.cz

IDS, Na Komořsku 2175/2a, 14300 Praha 4, Czech Republic

\begin{abstract}
Boháč J., Matějka K.: Communities of epigeic beetles in tree line from montane spruce forest to secondary meadow in the different stage of the forest decline in the area of Modrava (Bohemian forest, Czech Republic). Ekológia (Bratislava), Vol. 35, No. 4, p. 340-349, 2016.

Communities of epigeic beetles were studied using pitfall trapping on 10 plots with the different stage of decline and clear-cut plots without coarse woody debris. Species richness (number of all species, S), total species diversity as the Shannon-Wiener's index $(\mathrm{H})$ and equitability (e) were calculated in the DBreleve. The Ward's method of hierarchical agglomerative classification with Euclidean distance was used for the differentiation of the communities on the plots. Species data for this analyse were represented by logarithm-transformed activities $[\log (x+1)]$. The single-factor analysis of variance (ANOVA) was used for statistical testing of differences in structural parameters (e.g. species richness and diversity) amongst distinguished groups of the plots. The species diversity and activity differs on glades without trees and coarse woody debris. The highest number of species was found in clear-cut areas. The lowest number of species was found on the plots with the living forest particularly invaded by bark beetle. This fact is caused by the fact that the clear-cut plots are invaded by ubiquitous and anthropotolerant species with good migration possibilities. These species are adapted to habitats without trees and are able to leave even in the habitats with very sparse or without vegetation. Species living in the forest even under the bark beetle attack are often stenotopic and adapted to the forest microclimate (higher humidity and low average temperature). These species are very sensitive to great difference in the daily changes of microclimate. The study of beetle communities support the hypothesis that the keeping of dead tree stands on plots after bark beetle outbreak is better for biodiversity conservation than the cutting down of trees and the abolishment of stems.
\end{abstract}

Key words: epigeic beetle communities, montane spruce forest, forest decline, bark beetle outbreak, forest management.

\section{Introduction}

The most important challenge of the European forestry is a sustainable farming with both the feasible wood production and encouragement of non-production functions of forest includ- 
ing biodiversity (Lindenmayer et al., 2000). The majority of virginal forests in submontane and montane areas of the central Europe was cut down and changed on the spruce monocultures (Röhle, 1991; Pretsch, 1996; Vacek et al., 2009). These even-aged stands have the high productivity, but they are very sensitive to weather conditions (especially wind storms) and insects outbreaks (Rothe, Borchert, 2003).

In forests, competition between humans and saproxylic beetles has a long history (Grove, 2002). The European spruce bark beetle Ips typographus is considered to be the most serious pest species of mature spruce stands, mainly of Norway spruce (Picea abies), throughout Eurasia (Grodzki et al., 2004; Wermelinger, 2004). Amongst all types of forest damage from 1950 to 2000 in Europe, 2-9 million $\mathrm{m}^{3}$ of timber per year were destroyed by bark beetles, mainly Ips typographus (Schelhaas et al., 2003).

The preference of this species for mature spruce trees increases the economic impact of its damage. With regard to these facts, it is not surprising that most published research on bark beetles deals with damage and prevention of outbreaks in commercial forests (Eriksson et al., 2006; Hedgren, Schroeder, 2004; Reeve, 1997; Wermelinger, 2004). By contrast, some studies have pointed out the ecological value of I. typographus and other bark beetles as ecosystem engineers and the so-called keystone species, driving forest regeneration and producing snags and a rich patchiness in forest canopies (Jonášová, Prach, 2004; Martikainen et al., 1999; Müller et al., 2008). Apart from providing these ecosystem services, the arthropod complex associated with $I$. typographus, including more than 140 species (Weslien, 1992), is in itself a significant contribution to diversity. Boháč and Matějka (2009) approved that the diversity of epigeic and hemiedaphic beetles in the southern Šumava (Plechý Mt. region) is much higher in the dead tree stand with coarse woody debris in comparison with clear-cut areas with removed wood.

By assessing the habitat preference of species and differences in beetle communities on plots in the different stage of forest decline, this study addressed the following questions:

1. Does species activity and diversity alter on the plots with different stage of decline?

2. Do preferences of species for plots with different stages of spruce forest decline support the hypothesis that the keeping of dead tree stand on plots after bark beetle outbreak is better for biodiversity conservation than the cutting down of trees and the abolishment of stems?

\section{Material and methods}

Experimental plots

Ten plots were studied in the vicinity of Modrava in the National Nature Park Šumava. The characteristics of studied plots are described in Table 1. All plots are very similar concerning all characteristics with the exception of the past management and the stage of the spruce forest decline. We can designate three groups of experimental plots after the stage of the forest decline:

1. Group L - plots with actually living mature trees; many trees are currently invaded by bark beetle - see development of the individual plots in Matějka (2010).

2. Group D - plots with dead tree stand, wood is remaining in the ecosystem.

3. Group C - plots with damaged stands, which were clear-cut. 


\begin{tabular}{|c|c|c|c|c|c|c|c|c|c|c|c|}
\hline & $\begin{array}{l}\stackrel{\mathscr{J}}{0} \\
z \\
z\end{array}$ & 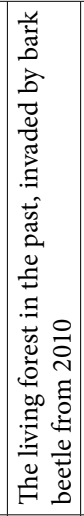 & 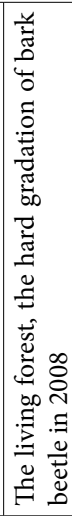 & 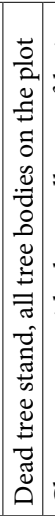 & 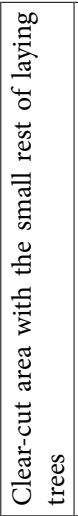 & 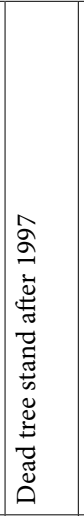 & 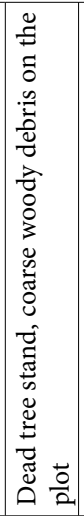 & 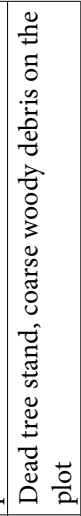 & 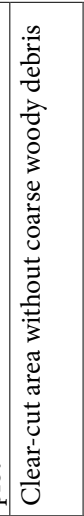 & 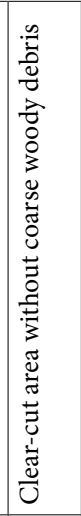 & 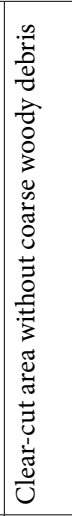 \\
\hline & 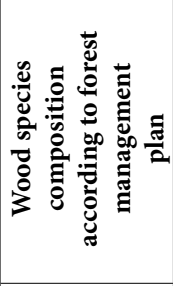 & 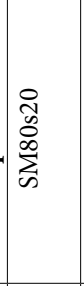 & $\underset{\sum}{\stackrel{8}{B}}$ & $\sum_{\infty}^{\circ}$ & 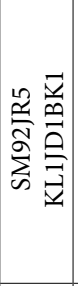 & 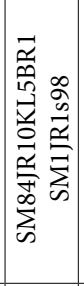 & 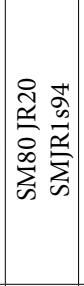 & 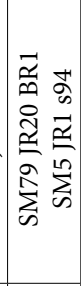 & 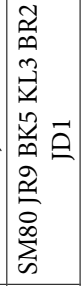 & 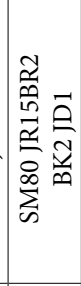 & 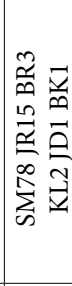 \\
\hline & 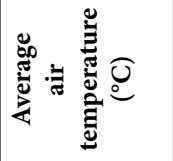 & $\hat{m}$ & $\stackrel{\circ}{+}$ & $\tilde{\dot{m}}$ & $\ddot{\dot{m}}$ & $\tilde{m}$ & $\stackrel{m}{n}$ & $\stackrel{n}{n}$ & $\ddot{m}$ & $\begin{array}{l}0 \\
\dot{r}\end{array}$ & $\stackrel{\ddot{n}}{\ddot{m}}$ \\
\hline & 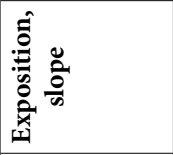 & \begin{tabular}{l}
8 \\
8 \\
$\dot{0}$ \\
\multicolumn{1}{|c|}{}
\end{tabular} & 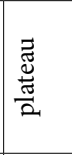 & 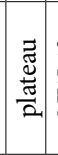 & 方 & in & $\begin{array}{l}\text { in } \\
3 \\
z\end{array}$ & $\begin{array}{l}\stackrel{0}{0} \\
\text { 至 } \\
\leftrightarrow\end{array}$ & $\begin{array}{l}\text { in } \\
\text { 裎 }\end{array}$ & 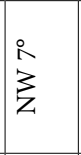 & $\begin{array}{l}\stackrel{\circ}{0} \\
\text { 荇 }\end{array}$ \\
\hline & 窇 & $\begin{array}{l}\stackrel{0}{0} \\
=\end{array}$ & $\underset{\underset{J}{\Xi}}{=}$ & 足 & $\stackrel{\text { త }}{=}$ & 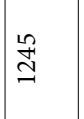 & $\begin{array}{l}\stackrel{\oplus}{]} \\
\text { In }\end{array}$ & 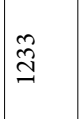 & 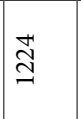 & $\stackrel{2}{\rightrightarrows}$ & $\stackrel{\stackrel{ }{I}}{\underset{I}{I}}$ \\
\hline & 蒂 & 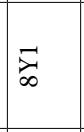 & $\begin{array}{l}\vec{\partial} \\
\frac{1}{\infty}\end{array}$ & $\begin{array}{l}= \\
\bar{n} \\
\infty \\
\infty\end{array}$ & $\begin{array}{l}\overrightarrow{\vec{a}} \\
\hat{n} \\
\infty \\
\infty \\
\vec{\infty} \\
\infty\end{array}$ & $\vec{\infty}$ & $\begin{array}{l}\overrightarrow{\vec{c}} \\
\infty \\
\stackrel{\infty}{\vec{\alpha}} \\
\infty\end{array}$ & $\begin{array}{l}\frac{1}{W} \\
\infty \\
\frac{\infty}{N} \\
\infty \\
\infty\end{array}$ & $\begin{array}{l}\overrightarrow{\vec{c}} \\
\frac{\infty}{\hat{\infty}} \\
\frac{\infty}{\infty}\end{array}$ & $\frac{\hat{y}}{\infty}$ & $\mid \begin{array}{l}\vec{a} \\
\frac{\infty}{\hat{N}} \\
\frac{2}{\infty}\end{array}$ \\
\hline & $\stackrel{\circ}{4}$ & ले & 8 & $\stackrel{\stackrel{m}{m}}{=}$ & $\infty$ & $n$ & 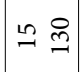 & $0 \stackrel{0}{0}$ & 0 & $\wedge$ & in \\
\hline & 营 & in & $m$ & in & - & -1 & $\neg \sigma$ & $\rightarrow+$ & - & - & - \\
\hline & 䔍 & 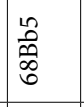 & 离 & 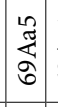 & 丞 & 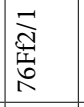 & 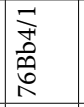 & 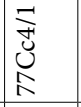 & 㞼 & $\overrightarrow{\tilde{O}}$ & 㞼 \\
\hline & 壹 & 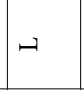 & $\mapsto$ & a & 0 & $\theta$ & $\theta$ & a & $u$ & 0 & 0 \\
\hline & $\frac{\overrightarrow{0}}{2}$ & $\bar{\Sigma}$ & $\stackrel{m}{\Sigma}$ & $\sum_{i}^{\pi}$ & $\sum^{0}$ & $\hat{\Sigma}$ & 胥 & 空 & 齐 & $\tilde{z}$ & 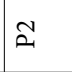 \\
\hline
\end{tabular}




\section{Sampling of beetles}

The method of pitfall trapping was used for the collection of epigeic beetles. A row of five plastic pitfall traps $(7 \mathrm{~cm}$ in diameter) was exposed in each locality from 20 June to 21 October 2010. Pitfall traps were filled with a mixture of ethylene glycol. The material from the traps was collected every month. The material was transported to the laboratory for the next processing and determination.

The degree of human impact was studied by finding the frequency of species of different ecological groups (Hůrka et al., 1996; Boháč, 1999; Boháč et al., 2008). The species were divided into groups as follows:

- Group r (relic species) includes species remaining from communities of past period, for example, species with arctic-alpine, boreo-montane and boreo-alpine occurrence, inhabiting mainly mountains and peat bogs or only occurring in remains of forests stands, which because of their high species diversity resemble recent climax forests.

- Group a (adaptable species) encompasses species of both natural and managed forests.

- Group e (generalists or ubiquitous species) comprises eurytopic species that successfully occupy deforested sites and are also found in areas strongly affected by man.

The method of ecological analysis of beetle communities (Boháč, 1999) was used for evaluating the community structure particularly. Frequency of ecological groups according to their relation to the naturalness of biotopes, preference of screened or opened habitats (or living in both) and hygropreference were used during this analysis. Increased influence of man was found to bring about an increase in the frequency of eurytopic species, species preferring opened habitats and species with lower moisture preferences.

\section{Statistical analysis}

Data were stored in the database DBrevele (Matějka, 2009). The basic processing of the data was made in this program. Data of the species activity (number of individuals caught in the five traps in the plot) from all months of sampling were summed for basic description of the investigated beetle communities. Species richness (number of all species, S), total species diversity as the Shannon-Wiener index (H) and equitability (e) were calculated in the DBreleve.

The Ward's method of hierarchical agglomerative classification (Ward, 1963) with Euclidean distance was used for the differentiation of the communities on the plots. Species data for this analyse were represented by logarithmtransformed activities $[\log (\mathrm{x}+1)]$.

The single-factor analysis of variance (ANOVA) was used to statistically test the differences in structural parameters (e.g. species richness and diversity) amongst distinguished groups of the plots.

\section{Results and discussion}

\section{Plots preferences (number of species and its activity)}

The number of species found in all studied plots was 89. The number of occurred species was different in studied plots (Table 2). The highest number of species was found in clear-cut areas (plots a0, ap2 and p2) - 34-36 species. The lower number of species (25) was occurred on clear-cut area and with the small rest of laying trees (M6) and on the plot with declining trees (M3). The lowest number of species was found on the plots with the living forest particularly invaded by bark beetle. This is due to the fact that the clear-cut plots are invaded by ubiquitous and anthropotolerant species with good migration possibilities. These species are adapted to habitats without trees and are able to leave even in the habitats with very sparse or without vegetation (e.g. Amara aenea, Poecilus versicolor, Bembidion lampros, Phyllotreta nemorum, Omalium caesum, O. rivulare, Byrrhus pilula).

Species living in the forest even under the bark beetle attack are often stenotopic and adapted to the forest microclimate (higher humidity and low average temperature). These 
$\mathrm{T} \mathrm{a} \mathrm{b}$ l e 2. The comparison of beetle species, its activity (number of individuals), diversity and equitability and the list of found species with its ecological characteristics on the studied plots. Ecological characteristics - breadth of the species ecological valence (e, eurytopic species; a, adaptable species; r, relic species) and habitat preference (s, screened habitats; o, open habitats) are marked by species names.

\begin{tabular}{|c|c|c|c|c|c|c|c|c|c|c|c|c|}
\hline Locality & & & a0 & ap2 & M6 & p2 & al2 & el1 & M3 & M7 & M1 & M4 \\
\hline Stand type & & & $\mathbf{C}$ & $\mathrm{C}$ & C & $\mathrm{C}$ & D & D & $\mathbf{L}$ & $\mathbf{D}$ & $\mathbf{L}$ & D \\
\hline Number of species & & & 36 & 34 & 25 & 34 & 21 & 18 & 25 & 24 & 15 & 30 \\
\hline Number of individuals & & & 196 & 165 & 126 & 175 & 106 & 71 & 101 & 86 & 85 & 127 \\
\hline Diversity & & & 4.44 & 4.46 & 3.89 & 4.40 & 3.91 & 3.80 & 4.19 & 4.29 & 3.65 & 4.57 \\
\hline Equitability & & & 0.86 & 0.88 & 0.84 & 0.87 & 0.89 & 0.91 & 0.90 & 0.94 & 0.93 & 0.93 \\
\hline Amara aenea (DeGeer, 1774) & $\mathrm{e}$ & o & 7 & 14 & 17 & 10 & & & & & & \\
\hline Poecilus versicolor (Sturm, 1824) & $\mathrm{e}$ & o & 4 & 4 & 14 & 6 & & & & & & \\
\hline Bembidion lampros (Herbst, 1784) & $\mathrm{e}$ & o & 4 & 6 & 5 & 8 & & & & & & \\
\hline Agriotes obscurus (Linnaeus, 1758) & e & o & 4 & 5 & 7 & 2 & & & & & & \\
\hline Calathus fuscus (Fabricius, 1792) & $\mathrm{e}$ & o & 2 & 4 & 2 & 4 & & & & & & \\
\hline Phyllotreta nemorum (Linnaeus, 1758) & $\mathrm{e}$ & o & 8 & 8 & 2 & 7 & & & & & & \\
\hline Omalium caesum Gravenhorst, 1802 & $\mathrm{e}$ & o & 4 & 5 & 3 & 8 & & & & & & \\
\hline Cantharis fusca Linnaeus, 1758 & $\mathrm{e}$ & o & 6 & 3 & 2 & 5 & & & & & & \\
\hline Omalium rivulare (Paykull, 1789) & $\mathrm{e}$ & o & 6 & 6 & 1 & 2 & & & & & & \\
\hline Byrrhus pilula (Linnaeus, 1758) & $\mathrm{e}$ & $\mathrm{o}$ & 5 & & 3 & 1 & & & & & & \\
\hline Eucnecosum brachypterum (Gravenhorst, 1802) & $\mathrm{r}$ & $\mathrm{o}$ & 1 & & 2 & 2 & & & & & & \\
\hline Tachinus signatus (Gravenhorst, 1802) & $\mathrm{e}$ & o & 5 & 8 & & 2 & & & & & & \\
\hline Pseudoophonus rufipes O.F. Müller, 1776 & $\mathrm{e}$ & o & 1 & 1 & & 2 & & & & & & \\
\hline Phyllotreta undulata (Kutschera, 1860) & $\mathrm{e}$ & o & 3 & 6 & & 1 & & & & & & \\
\hline Mycetoporus clavicornis (Stephens, 1832) & $\mathrm{a}$ & $\mathrm{o}$ & 12 & & & 9 & & & & & & \\
\hline Harpalus rubripes (Duftschmid, 1812) & $\mathrm{e}$ & o & 1 & & & 5 & & & & & & \\
\hline Tachyporus hypnorum ( Fabricius, 1775) & $\mathrm{e}$ & o & 1 & 2 & 4 & & & & & & & \\
\hline Loricera pilicornis (Fabricius, 1775) & e & $\mathrm{o}$ & 2 & 2 & 1 & & & & & & & \\
\hline Notiophilus biguttatus (Fabricius, 1779) & $\mathrm{e}$ & o & 2 & & 1 & & & & & & & \\
\hline Tachyporus chrysomelinus (Linnaeus, 1758) & $\mathrm{e}$ & o & 2 & & 2 & & & & & & & \\
\hline Anotylus rugosus (Fabricius, 1775 ) & & & 1 & & 1 & & & & & & & \\
\hline Sciodrepoides watsoni (Spence, 1815) & $\mathrm{e}$ & o & 35 & 7 & & & & & & & & \\
\hline Gabrius pennatus Sharp, 1910 & & & 2 & 1 & & & & & & & & \\
\hline Philonthus cognatus Stephens, 1832 & e & o & 5 & 3 & & & & & & & & \\
\hline Philonthus atratus (Gravenhorst, 1802) & e & o & 4 & & & & & & & & & \\
\hline Quedius paradisianus (Heer, 1839) & $\mathrm{a}$ & s & 2 & & & & & & & & & \\
\hline Coccinella septempunctata (Linnaeus, 1758) & $\mathrm{a}$ & $\mathrm{o}$ & & 2 & 2 & 3 & & & & & & \\
\hline Philonthus laevicollis (Lacordaire, 1853) & a & $\mathrm{s}$ & & 1 & 3 & 2 & & & & & & \\
\hline Lathrobium fulvipenne (Gravenhorst, 1806) & $\mathrm{e}$ & $\mathrm{o}$ & & 5 & 3 & & & & & & & \\
\hline Carabus violaceus Linnaeus, 1758 & $\mathrm{a}$ & so & & 2 & & 3 & & & & & & \\
\hline Agriotes sputator (Linnaeus, 1758) & & $\mathrm{O}$ & & 1 & & 2 & & & & & & \\
\hline Ocypus fuscatus (Gravenhorst, 1802) & $\mathrm{a}$ & o & & 1 & & 1 & & & & & & \\
\hline Oxypoda soror $\mathrm{C} . \mathrm{G}$. Thomson, 1855 & $a$ & o & & 1 & 1 & & & & & & & \\
\hline Sciodrepoides fumatus (Spence, 1815) & $\mathrm{e}$ & o & & 4 & & & & & & & & \\
\hline Cercyon sp. & $\mathrm{e}$ & o & & 1 & & & & & & & & \\
\hline Anotylus tetracarinatus (Block, 1799) & $\mathrm{e}$ & o & & 1 & & & & & & & & \\
\hline Platystetheus nitens (C. R. SAHLBERG, 1832) & e & o & & 1 & & & & & & & & \\
\hline Ontholestes murinus (Linnaeus, 1758) & & & & & 2 & & & & & & & \\
\hline Otiorhynchus singularis (Linnaeus, 1758) & $\mathrm{e}$ & o & & & & 4 & & & & & & \\
\hline Oeceoptoma thoracica (Linnaeus, 1758) & $\mathrm{e}$ & o & & & & 4 & & & & & & \\
\hline Melanotus rufipes (Herbst, 1784) & $\mathrm{e}$ & s & & & & 2 & & & & & & \\
\hline Otiorhynchus laevigatus Gyllenhal, 1834 & $\mathrm{e}$ & o & & & & 1 & & & & & & \\
\hline Nicrophorus vespilloides Herbst, 1807 & $\mathrm{e}$ & o & 8 & & & 7 & & & & & 6 & 4 \\
\hline Anthobium atrocephalum (Gyllenhal, 1827) & a & $\mathrm{s}$ & 1 & 5 & & 5 & 5 & 5 & & 5 & 5 & 3 \\
\hline Atheta fungi (Gravenhorst, 1806) & $\mathrm{e}$ & so & 12 & 33 & 25 & 41 & 2 & 2 & 3 & 2 & 4 & 5 \\
\hline Atheta crassicornis (Fabricius, 1792) & $\mathrm{e}$ & so & 28 & 8 & 16 & 11 & 3 & & & & 2 & 3 \\
\hline Amara communis (Panzer, 1797) & $\mathrm{e}$ & $\mathrm{o}$ & 2 & 7 & 5 & 6 & & & & & & 1 \\
\hline Harpalus affinis (Schrank, 1781) & $\mathrm{e}$ & o & 5 & 5 & & 3 & & & & & & 4 \\
\hline
\end{tabular}


$\mathrm{T}$ a b l e 2. The comparison of beetle species, its activity (number of individuals), diversity and equitability and the list of found species with its ecological characteristics on the studied plots. Ecological characteristics - breadth of the species ecological valence (e, eurytopic species; a, adaptable species; r, relic species) and habitat preference ( $\mathrm{s}$, screened habitats; o, open habitats) are marked by species names - continued.

\begin{tabular}{|c|c|c|c|c|c|c|c|c|c|c|c|c|}
\hline Locality & & & a0 & ap2 & M6 & p2 & al2 & el1 & M3 & M7 & M1 & M4 \\
\hline Stand type & & & $\mathrm{C}$ & $\mathrm{C}$ & $\mathrm{C}$ & C & D & D & $\mathbf{L}$ & D & L & D \\
\hline Number of species & & & 36 & 34 & 25 & 34 & 21 & 18 & 25 & 24 & 15 & 30 \\
\hline Number of individuals & & & 196 & 165 & 126 & 175 & 106 & 71 & 101 & 86 & 85 & 127 \\
\hline Diversity & & & 4.44 & 4.46 & 3.89 & 4.40 & 3.91 & 3.80 & 4.19 & 4.29 & 3.65 & 4.57 \\
\hline Equitability & & & 0.86 & 0.88 & 0.84 & 0.87 & 0.89 & 0.91 & 0.90 & 0.94 & 0.93 & 0.93 \\
\hline Hister unicolor Linnaeus, 1758 & $\mathrm{e}$ & o & 2 & & & & & & & & & 2 \\
\hline Mycetoporus lepidus (Gravenhorst, 1806) & $\mathrm{a}$ & $\mathrm{s}$ & 2 & & 2 & 2 & 1 & 1 & 1 & 1 & & \\
\hline Liparus glabrirostris Küster, 1849 & a & s & 2 & & & & 1 & & & & & \\
\hline Tachinus laticollis Gravenhorst, 1802 & $\mathrm{e}$ & o & 5 & & & & & & & 2 & & 1 \\
\hline Bryoporus rufus (Erichson, 1839) & $\mathrm{r}$ & o & & 2 & & 3 & 2 & & 2 & 2 & & 6 \\
\hline Pterostichus nigrita (Paykull, 1790) & $\mathrm{e}$ & $\mathrm{O}$ & & & & 1 & 1 & & & & 1 & \\
\hline Othius myrmecophilus Kiesenwetter, 1843 & $\mathrm{a}$ & s & & & & & 2 & & 2 & 2 & & 2 \\
\hline Pterostichus burmeisteri Heer, 1841 & $\mathrm{a}$ & $\mathrm{s}$ & & & & & 3 & 5 & 5 & 3 & & 7 \\
\hline Dinaraea aequata (Erichson, 1837) & $\mathrm{e}$ & s & & & & & 9 & 1 & 1 & 7 & & 3 \\
\hline Abax ovalis (Duftschmid, 1812) & a & s & & & & & 9 & 5 & 6 & 1 & 8 & 2 \\
\hline Carabus auronitens Fabricius, 1792 & $\mathrm{a}$ & $s$ & & & & & 14 & 10 & 10 & 9 & 13 & 9 \\
\hline Carabus linnaei Panzer, 1810 & $\mathrm{a}$ & $\mathrm{s}$ & & & & & 15 & 11 & 11 & 6 & 9 & 13 \\
\hline Carabus sylvestris Panzer, 1910 & $\mathrm{a}$ & s & & & & & 6 & 6 & 6 & 3 & 4 & 3 \\
\hline Anoplotrupes stercorosus (Hartmann in L. Scriba, 1791) & a & s & & & & & 12 & 6 & 7 & 8 & 8 & \\
\hline Anthobium melanocephalum (Illiger, 1794) & a & $\mathrm{s}$ & & & & & 6 & 4 & 5 & 5 & 6 & \\
\hline Domene scabricollis (Erichson, 1840) & $\mathrm{a}$ & $\mathrm{s}$ & & & & & 2 & & 13 & 2 & 10 & \\
\hline Leptusa pulchella (Mannerheim, 1830) & $\mathrm{a}$ & s & & & & & 7 & 5 & 7 & 4 & & \\
\hline Trechus pulchellus Putzeys, 1846 & $\mathrm{a}$ & $\mathrm{s}$ & & & & & 1 & 2 & 1 & 1 & & \\
\hline Quedius cincticollis Kraatz, 1857 & a & $s$ & & & & & 3 & & 2 & 4 & & \\
\hline Philonthus rotundicollis (Ménétriés, 1832) & $\mathrm{a}$ & $\mathrm{s}$ & & & & & 2 & & & & & \\
\hline Pterostichus oblongopunctatus (Fabricius, 1787) & $\mathrm{a}$ & s & & & & & & 2 & 5 & 3 & & 5 \\
\hline Mycetoporus erichsonanus Fagel, 1965 & $\mathrm{r}$ & $\mathrm{s}$ & & & & & & 2 & 1 & & & 4 \\
\hline Ips typographus (Linnaeus, 1758) & $\mathrm{e}$ & o & & & & & & 1 & & & & 2 \\
\hline Rhizophagus dispar (Paykull, 1800) & $\mathrm{a}$ & s & & & & & & 2 & & & & 4 \\
\hline Atomaria sp. & & & & & & & & 1 & & & 1 & \\
\hline Platynus assimilis Paykull, 1790 & $\mathrm{a}$ & $\mathrm{s}$ & & & & & & & 2 & 3 & & 12 \\
\hline Acrotrichis sp. & a & $\mathrm{s}$ & & & & & & & 2 & 2 & 4 & 4 \\
\hline Gaurotes virginea (Linnaeus, 1758) & $\mathrm{a}$ & so & & & & & & & 1 & & 4 & 4 \\
\hline Othius angustatus Stephens, 1833 & $\mathrm{a}$ & $\mathrm{O}$ & & & & & & & 1 & 1 & & \\
\hline Atrecus longiceps (Fauvel, 1873) & $\mathrm{r}$ & $\mathrm{s}$ & & & & & & & 1 & & & \\
\hline Quedius boops (Gravenhorst, 1802) & $\mathrm{e}$ & so & & & & & & & 4 & & & \\
\hline Phyllobius calcaratus Schoenherr, 1826 & $\mathrm{e}$ & 0 & & & & & & & 2 & & & \\
\hline Liogluta granigera (Kiesenwetter, 1850) & $\mathrm{a}$ & s & & & & & & & & 8 & & 2 \\
\hline Cychrus caraboides (Linnaeus, 1758) & $\mathrm{a}$ & so & & & & & & & & 2 & & \\
\hline Quedius plagiatus Mannerheim, 1843 & $\mathrm{a}$ & $\mathrm{s}$ & & & & & & & & & & 11 \\
\hline Hylastes cunicularius Erichson, 1839 & $\mathrm{e}$ & o & & & & & & & & & & 4 \\
\hline Carabus glabratus Paykull, 1790 & $\mathrm{a}$ & so & & & & & & & & & & 2 \\
\hline Dexiogya corticina (Erichson, 1837) & a & $\mathrm{s}$ & & & & & & & & & & 2 \\
\hline Hylobius abietis (Linnaeus, 1758) & $\mathrm{a}$ & $s$ & & & & & & & & & & 2 \\
\hline Rhizophagus grandis (Gyllenhal, 1827) & $\mathrm{a}$ & $\mathrm{s}$ & & & & & & & & & & 1 \\
\hline
\end{tabular}

species are very sensitive to great difference in the daily changes in microclimate (Othius myrmecophilus, Pterostichus burmeisteri, Dinaraea aequata, Abax ovalis, Carabus auronitens, C. linnaei, C. sylvestris, Anthobium melanocephalum, Domene scabricollis, Trechus pulchellus, Leptusa pulchella, Quedius plagiatus). 


\section{Classification of the studied plots}

Classification of beetle communities on the studied plots (Fig. 1) shows two main groups of the plots. It is clear that the clear-cut plots with removed wood are situated in the separated cluster A. Experimental plots with living trees (L) and with declined stands are situated in the second cluster B.

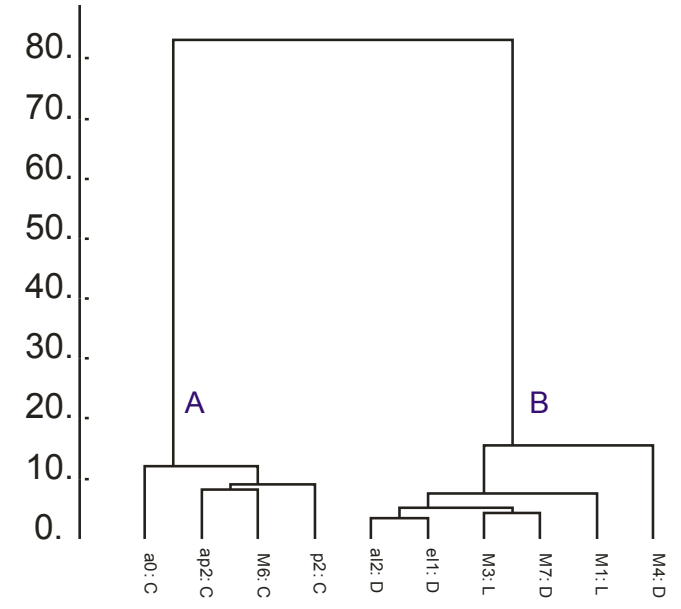

Fig. 1. Classification of beetle communities on studied plots (Ward's method with quadrate of Euclidean distance). Transformed data of the activity (number of specimen) in pitfall traps were used in all months (June-October).

Number of species, activity and the total species diversity are higher on the plots of the group A (Figs 2-5). This fact is caused by the higher activity of eurytopic species, which are able to quickly occupy deforested areas. Some forest species are still able to survive on these plots. The equitability of beetle communities is higher on plots with living forest or forest with different stage of decline on the other site.

Distribution of ecological groups of beetles on studied plots

The species can be separated into three groups after their presence or absence in the plots representing classification groups A and B (Table 2).

Species typical for the first classification group A can be identified as r-strategists after Pianka (1970). These species are indicated as eurytopic species that successfully occupy deforested sites and are also found in areas strongly affected by man. The surprise

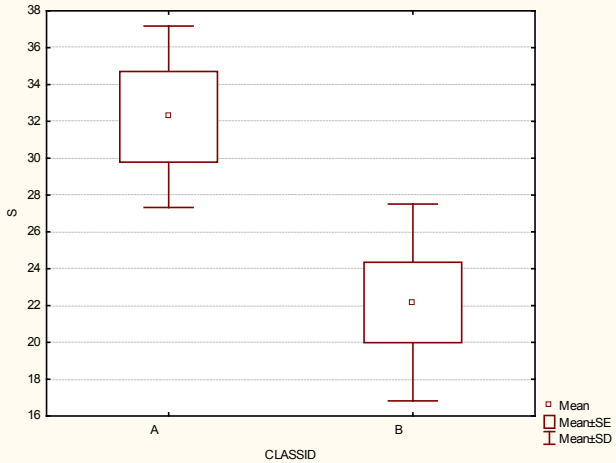

Fig. 2. Number of species, ANOVA: $\mathrm{p}=0.016835$.

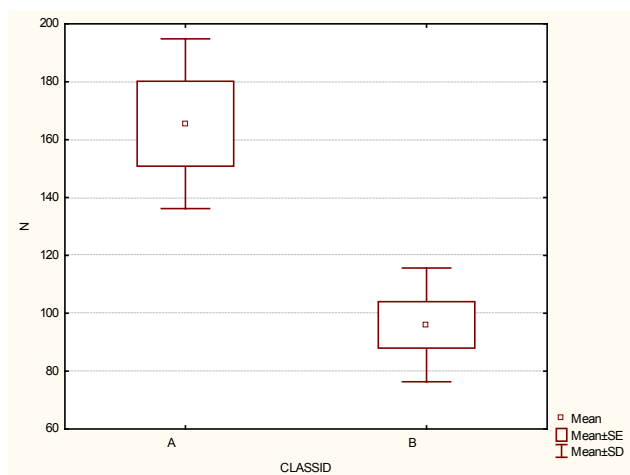

Fig. 3. Number of specimen, ANOVA: $\mathrm{p}=0.001916$. 


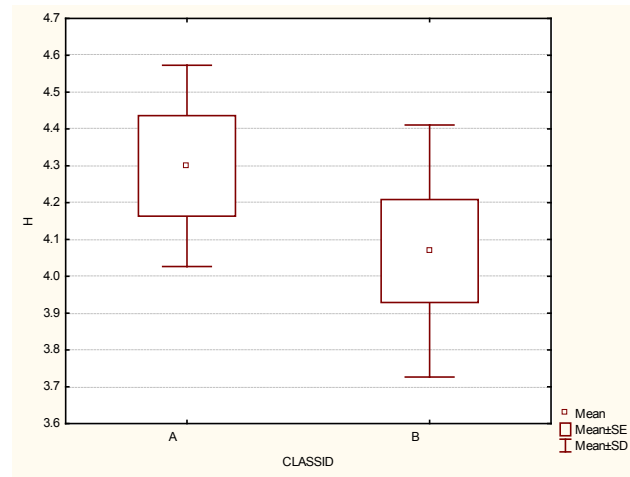

Fig. 4. Shannon-Wiener's index of species diversity, ANOVA: $\mathrm{p}=0.292833$.

is the presence of relic species Eucnecosum brachypterum. This species prefer montane meadows, stony debris, snow fields and plots affected by avalanches. It is possible that plots with fresh and dead trees is suitable for this species.

Species typical for the classification group B covers with ones of the narrower ecological valence. This group includes all three ecological groups after Boháč (1999) and Hurka et al. (1996). The minority represents very rare montane species (2 species). Staphylinid beetle Atrecus longiceps (Fig. 6) is named as indicators of rests of virginal montane forests (Müller et al., 2005). The majority of this beetle species belongs to the group of adaptable species living in both natural and managed forests. Small portion of species belongs to eurytopic species (previous group). These species are adapted to living in decaying substances (Dinaraea aequata), phytophagous species with good flying possibilities migrated to forest from cutovers (Phyllobius calcaratus) or living in dead wood (Hylastes cunicularius)

Neutral species cover different preference of habitats. Some relic montane species were found preferred montane meadows (Bryoporus rufus). The proportion of eurytopic species and adaptable species is approximately similar.

\section{Seasonal dynamics of the beetle community}

Changes in total epigeic beetle activity during the year 2010 (Fig. 6) shows the highest activity in June and July. The activity is decreasing from July to September rapidly. The small elevation was observed during October. We can see the similar character of the dynamics 


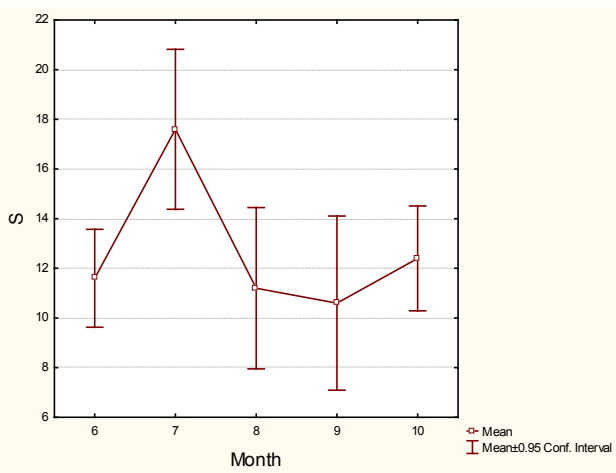

Fig. 7. Changes in species richness of epigeic beetle during the year 2010 .

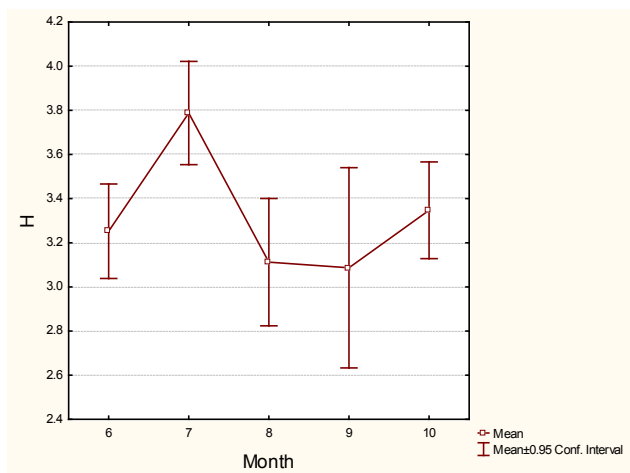

Fig. 8. Changes in total species diversity of epigeic beetle (Shannon-Wiener's index) during the year 2010.

by monitoring the changes in species richness (Fig. 7) and total species diversity (ShannonWiener's index) of epigeic beetles during the year (Fig. 8).

\section{Summary}

Communities of epigeic beetles were studied on 10 plots with different stage of decline and clear-cut plots without coarse woody debris. The species diversity and activity differs on glades without trees and coarse woody debris. The study of beetle communities supports the hypothesis that the keeping of dead tree stands on plots after bark beetle outbreak is better for biodiversity conservation than the cutting down of trees and the abolishment of stems.

\section{References}

Boháč, J. (1999). Staphylinid beetles as bioindicators. Agric. Ecosyst. Environ., 74, 357-372. DOI: 10.1016/S01678809(99)00043-2.

Boháč, J., Matějíček, J. \& Rous R. (2008). Check-list of staphylinid Beetles (Coleoptera, Staphylinidae) of the Czech Republic and the division of species according to their ecological characteristics and sensitivity to human influence. Časopis Slezského Muzea Opava (A), 56, 227-276.

Boháč, J. \& Matějka K. (2009). Sledování epigeických brouků na výškovém transektu na Plechém (Šumava) v roce 2009. Závěrečná zpráva projektu „Biodiverzita Šumavy a Krkonoš. www.infodatasys.cz.

Eriksson, M., Lilja, S. \& Roininen H. (2006): Dead wood creation and restoration burning: implications for bark beetles and beetle induced tree deaths. For. Ecol. Manag., 231, 205-213. DOI: 10.1016/j.foreco.2006.05.050.

Grodzki, W., McManus, M., Knizek, M., Meshkova, V., Mihalciuc, V., Novotny, J., Turcani, M. \& Slobodyan Y. (2004). Occurrence of spruce bark beetles in forest stands at different levels of air pollution stress. Environ. Pollut., 130, 73-83. DOI: 10.1016/j.envpol.2003.10.022.

Grove, S. (2002). Trunk window trapping: an effective technique for sampling tropical saproxylic insects. Mem. Queensl. Mus., 46, 149-160.

Hedgren, P.O. \& Schroeder L.M. (2004). Reproductive success of the spruce bark beetle Ips typographus (L.) and occurrence of associated species: a comparison between standing beetle-killed trees and cut trees. For. Ecol. Manag., 203, 241-250. DOI: 10.1016/j.foreco.2004.07.055.

Hůrka, K., Veselý, P. \& Farkač J. (1996). Využití stř̌evlíkovitých (Coleoptera: Carabidae) k indikaci kvality prostředí. Klapalekiana, 32, 15-26.

Jonášová, M. \& Prach K. (2004). Central-European mountain spruce (Picea abies (L) Karst.) forests: regeneration of 
tree species after a bark beetle outbreak. Ecological Engineering, 23, 15-27. DOI: 10.1016/j.ecoleng.2004.06.010.

Lindenmayer, D.B., Margules, C.R. \& Botkin D.B. (2000). Indicators of biodiversity for ecologically sustainable forest management. Conserv. Biol., 14, 941-950. DOI: 10.1046/j.1523-1739.2000.98533.x.

Martikainen, P., Siitonen, J., Kaila, L., Puntilla, P. \& Rauh J. (1999). Bark beetles (Coleoptera, Scolytidae) and associated beetle species in mature managed and old-growth boreal forests in southern Finland. For. Ecol. Manag., 116, 233-245. DOI: 10.1016/S0378-1127(98)00462-9.

Matějka, K. (2009). Nápověda k programu DBreleve. URL: http://www.infodatasys.cz/software/hlp_dbreleve/ dbreleve.htm

Matějka, K. (2010). Vývoj vegetace na TVP Šumava. URL: http://www.infodatasys.cz/sumava/vegdyn_cz.htm

Müller, J., Bussler, H., Bense, U., Brustel, H., Fletchner, G., Fowles, A., Kahlen, M., Möller, G., Mühle, H., Schmidl, J. \& Zabransky P. (2005). Urwald relict species - Saproxylic beetles indicating structural qualities and habitat tradition. Waldoekologie, 2, 106-113.

Pianka, E.R. (1970). On $\mathrm{r}$ and K selection. Am. Nat., 104, 592-597. DOI: 10.1086/282697.

Pretsch, H. (1996). Growth trends of forests in Southern Germany. In H. Spiecker, K. Mielikäinen, M. Köhl \& J.P. Skovsgaard (Eds.), Growth trends in european forests (pp. 107-131). European forest institute report No. 5. Berlin, Heridelberg, New York: Springer.

Reeve, J.D. (1997). Predation and bark beetle dynamics. Oecologia, 112, 48-54. DOI: 10.1007/s004420050282.

Röhle, H. (1991). Entwicklung der wichtigsten ertragskundlichen Kenngrössen des Fichtenwalbestandes Högwald in der 6-jährigen Beobachtungs periode 1083-1988. Forstwissenschaftlichen Forschung H, 39, 30-34.

Rothe, A. \& Borchert H. (2003): Der Wald von morgen. Eine Naturbilanz über 25 Jahre naturnahe Forstwirtschaft im Bayerischen Staatswald. LWF 39. Munich: Bayerische Landesanstalt für Wald und Forstwirtschaft.

Schelhaas, M.J., Nabuurs, G.J. \& Schuck A. (2003). Natural disturbances in the European forests in the 19th and 20th centuries. Global Change Biology, 9, 1620-1633. DOI: 10.1046/j.1365-2486.2003.00684.x.

Vacek, S., Krejčí, F., Remeš, J., Ulbrichová, I., Svoboda, J.M., Zatloukal, V., Poustka, V., Simon, J., Minx, T., Bílek, L., Bednařík, J., Štícha, V., Malík, K., Jankovský, L., Vojtíšek, R., Baláš, M., Kozel, J., Malík, V. \& Matějka K. (2009). Struktura a vývoj porostů TVP, struktura lesa v přiblížení přirozené skladbě porostů. In S. Vacek \& F. Krejčí (Eds.), Lesní ekosystémy v Národním parku Šumava (pp. 135-320). Kostelec nad Černými lesy: Lesnická práce, s.r.o.

Ward, J.H. (1963): Hierarchical grouping to optimize an objective function. Journal of the American Statistical Association, 58(301), 236-244.

Wermelinger, B., Flückinger, P.F., Obrist, M.K. \& Duelli P. (2007). Horizontal and vertical distribution of saproxylic beetles (Col., Buprestidae, Cerambycidae, Scolytinae) across sections of forest edges. J. Appl. Entomol., 131, 104-114. DOI: 10.1111/j.1439-0418.2006.01128.x.

Weslien, J. (1992). The arthropod complex associated with Ips typographus (L.) (Coleoptera, Scolytidae): species composition, phenology and impact on bark beetle productivity. Entomol. Fenn., 3, 205-213. 